ARTICLE

\title{
Atomic-resolution three-dimensional hydration structures on a heterogeneously charged surface
}

\author{
Kenichi Umeda ${ }^{1,2}$, Lidija Zivanovic ${ }^{3}$, Kei Kobayashi ${ }^{1,4}$, Juha Ritala ${ }^{3}$, Hiroaki Kominami ${ }^{1}$, Peter Spijker (1) ${ }^{3}$,
} Adam S. Foster (iD ${ }^{3,5} \&$ Hirofumi Yamada ${ }^{1}$

Local hydration structures at the solid-liquid interface around boundary edges on heterostructures are key to an atomic-level understanding of various physical, chemical and biological processes. Recently, we succeeded in visualising atomic-scale three-dimensional hydration structures by using ultra-low noise frequency-modulation atomic force microscopy. However, the time-consuming three-dimensional-map measurements on uneven heterogeneous surfaces have not been achieved due to experimental difficulties, to the best of our knowledge. Here, we report the local hydration structures formed on a heterogeneously charged phyllosilicate surface using a recently established fast and nondestructive acquisition protocol. We discover intermediate regions formed at step edges of the charged surface. By combining with molecular dynamics simulations, we reveal that the distinct structural hydrations are hard to observe in these regions, unlike the charged surface regions, possibly due to the depletion of ions at the edges. Our methodology and findings could be crucial for the exploration of further functionalities.

\footnotetext{
${ }^{1}$ Department of Electronic Science and Engineering, Kyoto University, Katsura, Nishikyo, Kyoto 615-8510, Japan. ${ }^{2}$ Department of Advanced Material Science, The University of Tokyo, Kashiwa, Chiba 277-8561, Japan. ${ }^{3}$ COMP Centre of Excellence, Department of Applied Physics, Aalto University, Helsinki FI-00076, Finland. ${ }^{4}$ The Hakubi Center for Advanced Research, Kyoto University, Katsura, Nishikyo, Kyoto 615-8520, Japan. ${ }^{5}$ Division of Electrical Engineering and Computer Science, Kanazawa University, Kanazawa, 920-1192, Japan. Correspondence and requests for materials should be addressed to A.S.F. (email: adam.foster@aalto.fi) or to H.Y. (email: h-yamada@kuee.kyoto-u.ac.jp)
} 
A long with growing concern over energy and environmental issues, functional materials and devices utilising the catalytic, electrochemical and even biological processes at the solid-liquid interface have gained great attention ${ }^{1-7}$. Heterostructures, nano-clusters, edges and defects are particularly crucial in these processes, because these structures often amplify the reaction efficiencies due to quantum effects, low-coordinated atoms or concentrated electric fields ${ }^{2-4,7}$.

Since they are always accompanied by local hydration changes reflecting the local structural and chemical changes of adsorption/ surface species, understanding the local hydration structures at such reactive places is also essential. The hydration structures in complicated biological crystal samples have been extensively studied by $\mathrm{X}$-ray/neutron crystallography, nuclear magnetic resonance and cryoelecton microscopy ${ }^{8}, 9$. However, all of these measurements require samples in a crystalline state, of which the hydrations at such local structures are averaged out and also often differ from those in liquid environments. Although the hydration structures in liquid environments have been extensively studied by X-ray/neutron reflectivity ${ }^{10}$ and surface force measurements ${ }^{11}$, their lateral structure has hardly ever been observed.

Recently, we have opened the door to atomic-scale threedimensional (3D) mapping of the local hydration structures by ultra-low noise frequency-modulation atomic force microscopy $(\mathrm{FM}-\mathrm{AFM})^{12}, 13$, which has been applied to various homogeneous samples ${ }^{12-18}$. Moreover, with the support from theoretical molecular dynamics (MD) simulations, deeper molecular detail has been achieved ${ }^{17,19-23}$. However, the time-consuming atomicscale $3 \mathrm{D}$ hydration measurements on the uneven heterogeneous surfaces have never been achieved, to the best of our knowledge, because these experiments are extremely difficult without any deformation of the surface structures by the tip. Therefore, no one has yet observed atomic-scale local hydration structures between surfaces of different compositions as far as we know. Furthermore, the relationship between the local hydrations and surface properties on heterogeneous systems was theoretically discussed $^{24}$, but validation against experiments is also necessary. Previously, we established a fast and nondestructive acquisition protocol of 3D map measurements on uneven heterogeneous DNA samples without damaging the molecules ${ }^{25}$ (see 'Methods' section for details).

In this study, we show the local hydration structure around a domain boundary between heterogeneous areas with different structures and charges on a phyllosilicate surface. Molecular-scale hydration structures on the terraces are visualised by 3D FMAFM in aqueous solution, which are reproduced by the MD simulations. The simulations show different ion adsorption behaviours on oppositely charged terraces, which are reflected in the long-range electrostatic force in the experiment. We reveal the intermediate regions near the step edge on the positively charged terrace by 3D FM-AFM, presumably caused by the ion depletion near the step edge, which is predicted by the MD simulations.

\section{Results}

Structure of a heterogeneously charged surface. Clinochlore, $(\mathrm{Mg}, \mathrm{Fe})_{5} \mathrm{Al}\left(\mathrm{Si}_{3} \mathrm{Al}\right) \mathrm{O}_{10}(\mathrm{OH})_{8}$, belongs to the chlorite group of phyllosilicate materials, which are ubiquitous minerals existing over wide temperature and pressure ranges ${ }^{26-29}$ (see Supplementary Methods for determination of the composition and orientation of the crystal used in this study). It is composed of alternating structures of a tetrahedral-octahedral-tetrahedral $(2: 1)$ negatively charged talc-like $(\mathrm{T})$ layer of $\left[(\mathrm{Mg}, \mathrm{Fe})_{3}\left(\mathrm{Si}_{3} \mathrm{Al}\right)\right.$ $\left.\mathrm{O}_{10}(\mathrm{OH})_{2}\right]^{-}$and the positively charged brucite-like (B) layer of $\left[(\mathrm{Mg}, \mathrm{Fe})_{2} \mathrm{Al}(\mathrm{OH})_{6}\right]^{+}$(Fig. 1a and Supplementary Movie 1). Note that each layer is equivalent to phlogopite mica or hydrotalcite excluding the intercalating ions ${ }^{24}$. Upon cleavage of the crystal, this unique character of clinochlore allows us to investigate the step edges where the $\mathrm{T}$ and $\mathrm{B}$ layers alternate in great detail with both the 3D FM-AFM and MD simulations.

After cleavage of the sample, we obtained a large-scale topographic image of the clinochlore (001) surface in a $100 \mathrm{mM}$ $\mathrm{KCl}$ aqueous solution (Fig. 1b). In this image, we see island structures with stripe-like patterns running along the $[\overline{3} 10]$ direction, with the typical size of $100 \times 10 \mathrm{~nm}^{2}$. From the crosssectional line profile along P-Q (Fig. 1c), we deduced the island

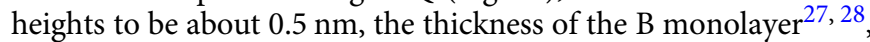
meaning that the higher islands are the B layer and the other regions are the $\mathrm{T}$ layer. Note that some islands in Fig. $1 \mathrm{~b}$ have characteristic triangular shapes and step directions similar to the side of the drawn blue triangle, as previously reported ${ }^{29,30}$.

In order to investigate the clinochlore island structure, we obtained an atomic-scale topographic image (Fig. 1d). On the T layer, atomic contrast is clearly visible, while the $\mathrm{B}$ layer seems to be composed of central and peripheral regions, indicated as $B_{I}$ and $B_{I I}$, respectively. The $B_{\text {II }}$ region has a lower height of $0.3 \mathrm{~nm}$ instead of $0.5 \mathrm{~nm}$ for the $B_{I}$ region. Figure 1e, f show enlarged atomic resolution images of the $T$ and $B_{I}$ regions, respectively. We observed honeycomb-like patterns with a $0.53-\mathrm{nm}$ spacing on the $\mathrm{T}$ regions and a hexagonal lattice with a $0.31-\mathrm{nm}$ spacing on the $B_{I}$ regions. In the $B_{I I}$ regions, a similar hexagonal pattern was observed, but was less clear than on $\mathrm{B}_{\mathrm{I}}$ (Supplementary Fig. 1).

Comparison between the experimental and theoretical data. In Fig. $2 \mathrm{a}, \mathrm{b}$, we show representations of the $3 \mathrm{D}$ force map obtained across an area including the $\mathrm{T}, \mathrm{B}_{\mathrm{II}}$ and $\mathrm{B}_{\mathrm{I}}$ regions (Supplementary Movie 2) and the equivalent MD simulation (Supplementary Note 1 and Supplementary Movie 3), respectively. In order to better understand the nature of the $\mathrm{B}_{\mathrm{II}}$ region, we first focus in detail on the hydration structures found on the $\mathrm{B}_{\mathrm{I}}$ and $\mathrm{T}$ regions. Previous studies have already shown that the force profiles or maps obtained by AFM gave good qualitative agreement with the density distributions of the water molecules determined by the Xray reflectivity or predicted by the MD simulations ${ }^{13}, 14,17,23,31-$ 35. Specifically, it has been reported in some papers that AFM force maps were well reproduced by free-energy calculations ${ }^{17,32}$. We first analysed the hydration layers on the T region in the same manner as in ref. ${ }^{13}$, where we assumed that the hydration force maxima correspond to the density maxima. For the $\mathrm{T}$ region, we could clearly distinguish two hydration layers. In Fig. $2 c$, the honeycomb-like pattern of the first hydration layer (closest to the surface) as observed in the AFM experiments is shown, and a similar pattern (Fig. 2e) is seen in the $2 \mathrm{D}$ water density distribution of the first higher layer. The second hydration layer above the $\mathrm{T}$ region follows much more like a dot-like pattern in both the experiment and simulation (Fig. 2d, f). We can see highly localised spots in the honeycomb hollows in the $2 \mathrm{D}$ water density distribution of the first higher layer (Fig. 2e). These dots are seen only in some honeycomb hollows because of the limited simulation time and they correspond to the tails of the adsorbed first lower layer, mainly contributed by the water molecules adsorbed in the honeycomb hollow. These are related to counter cations resident at the sites, but would be averaged out, and appear much fainter, if the simulations could be run long enough to obtain full sampling of the ion mobility. In the case of the $B_{I}$ region, atomic-scale patterns with the same lattice constant are observed in both the experiment (Fig. 2g, h) and simulation (Fig. 2i, j). While the $2 \mathrm{D}$ water density distributions show bright and dark dot patterns for the first (Fig. 2i) and the second (Fig. 2j) hydration layers, respectively, the patterns observed in the experiment are somewhat ambiguous. It is difficult to judge if 

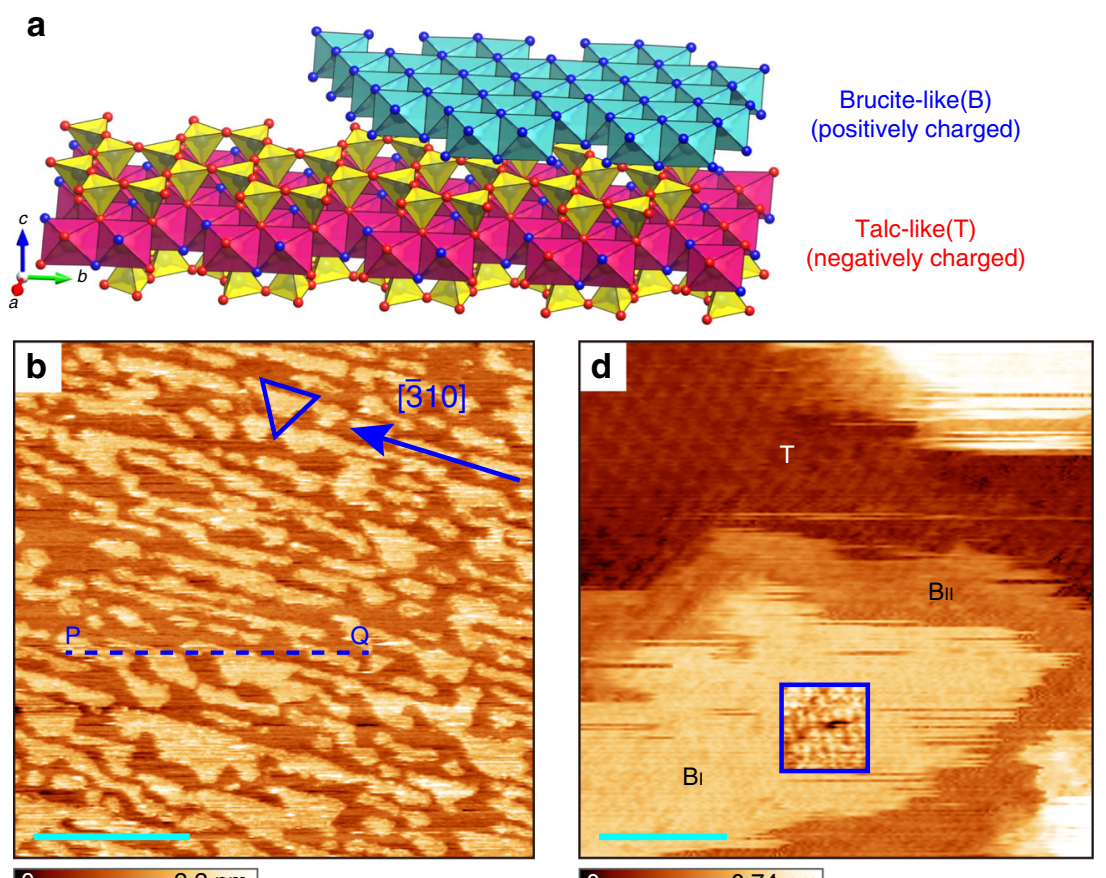

$0 \quad 2.2 \mathrm{~nm}$
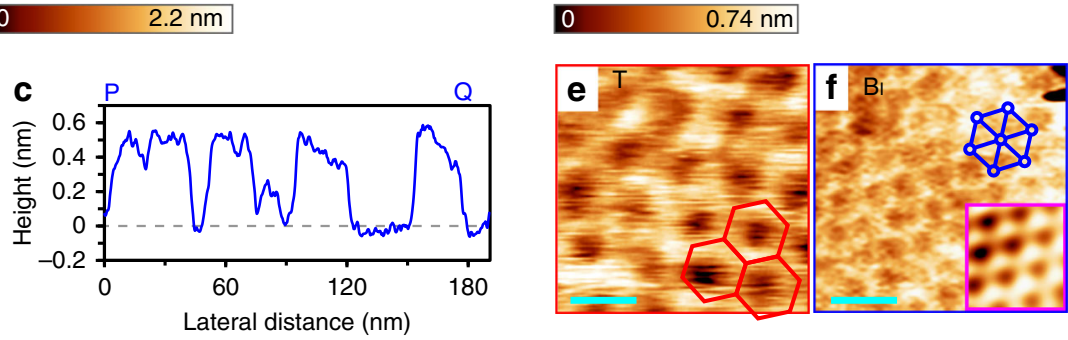

Fig. 1 FM-AFM images of clinochlore. a Crystal structure of clinochlore crystal, which is composed of negatively charged talc-like (T) and positively charged brucite-like (B) layers. The figure was drawn using VESTA ${ }^{42}$. b Topographic image of the clinochlore (001) surface. The triangle shows the characteristic shapes due to the partial dissolution of the B layer ${ }^{29}$. c Cross-sectional profile along the P-Q line indicated in $\mathbf{b}$. $\mathbf{d}$ Atomic-resolution image of the clinochlore (001) surface showing that the $B$ island has two regions $\left(B_{\mid}\right.$and $\left.B_{\|}\right)$with different heights. In the blue square, the contrast was enhanced and smoothed by a Gaussian filter. e, $\mathbf{f}$ Magnified atomic-resolution images taken on the $T$ region (e), and $B_{1}$ region (f), with the inset of an FFT-filtered image. All of the images were acquired with $\Delta f \sim+300 \mathrm{~Hz}$. Scale bars: $100 \mathrm{~nm}(\mathbf{b}), 3 \mathrm{~nm}(\mathbf{d})$, and $0.5 \mathrm{~nm}(\mathbf{e}, \mathbf{f})$

they are the same as those predicted from the simulation or even inverted because the contrast varies as a function of the tip height and conditions ${ }^{36}$. Since the lattice spacing of the $\mathrm{B}$ layer is smaller than that of the $\mathrm{T}$ layer and comparable to the size of the water molecule, it is plausible that the hydrated water molecules other than that on the tip apex play a role in the contrast formation and cause the patterns to be ambiguous or possibly inverted. As we show later, the anions form a honeycomb-like pattern at the same height of the first hydration layer on the B layer, which is the inverted pattern of the water (oxygen) distribution and may play a role in this observation.

From both the experimental and theoretical data, we extracted force and density maps perpendicular to the clinochlore surface along the lines indicated in Fig. 2c, e, g, i. On each of these four maps, we can identify the respective lateral spacing, which is 0.53 $\mathrm{nm}$ for the $\mathrm{T}$ region (Fig. $2 \mathrm{k}, \mathrm{l}$ ) and $0.31 \mathrm{~nm}$ for the $\mathrm{B}_{\mathrm{I}}$ region (Fig. $2 \mathrm{~m}, \mathrm{n}$ ).

From the discussion above, we found that the apparent surfaces (tip trajectories) are the first layers instead of the bare mineral surface, and the molecular-scale patterns observed in the experiment were in good agreement with the $2 \mathrm{D}$ water density distributions in the first and second hydration layers. It should be mentioned that when we set the threshold values higher, the tip change was often induced by a strong interaction force and the continuity of the consecutively recorded force curves was deteriorated. These results demonstrate that the combination of liquid-3D FM-AFM and MD simulation is useful for illuminating the details of $3 \mathrm{D}$ water distributions.

Quantitative analysis of force curves. For more quantitative analysis, we also constructed averaged force curves (measured at 200 randomly selected pixels) for both the $\mathrm{T}$ and $\mathrm{B}_{\mathrm{I}}$ regions from the experiment (Fig. 3a, b). While the similar oscillatory features are obvious in both cases, the repulsive and attractive background forces are seen on the $\mathrm{T}$ and $\mathrm{B}_{\mathrm{I}}$ regions, respectively. We fitted these background forces by exponential functions with decay distances of 0.15 and $0.9 \mathrm{~nm}$ for the $T$ and $B_{I}$ regions, respectively. The decay distance of $0.9 \mathrm{~nm}$ on the $B_{I}$ region is almost equivalent to the Debye length in the $100 \mathrm{mM} \mathrm{KCl}$ solution, meaning that this force component is likely the long-range electric doublelayer force ${ }^{25}$. The reason why this force was hardly observed on the $\mathrm{T}$ region can be explained by the fact that most of the surfacenegative charges on the $\mathrm{T}$ layer are neutralised by the coadsorption of the proton and cation in the solution of $\mathrm{pH} 5.7^{37}$. This strong adsorption of cations on the $\mathrm{T}$ layer is also seen in our simulations, which will be shown later. For this reason, while the structural charge density of the T (-) and B (+) layers is the same, $\pm 0.32 \mathrm{C} / \mathrm{m}^{2}$, the apparent surface charge densities are different, -0.015 and $+0.32 \mathrm{C} / \mathrm{m}^{2}$, respectively ${ }^{37}, 38$. We estimated that the 
a
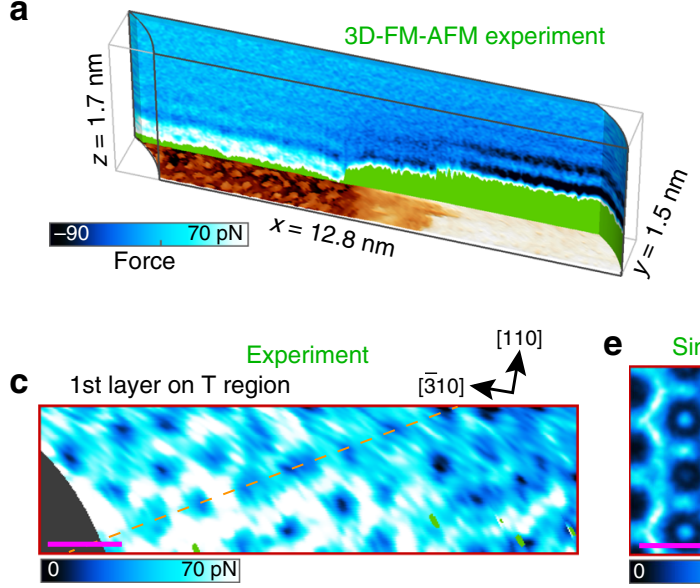

d 2nd layer on T region

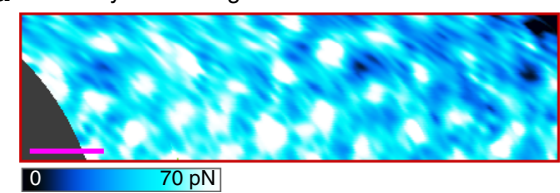

Experiment

k Perpendicular map on T region

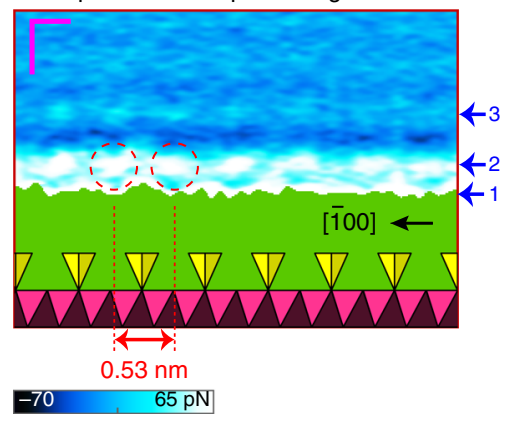

e
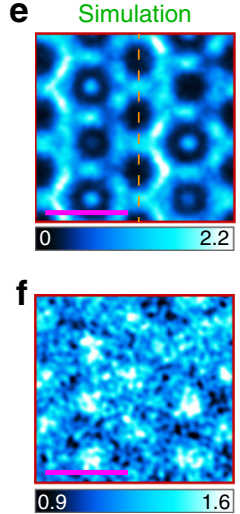

b

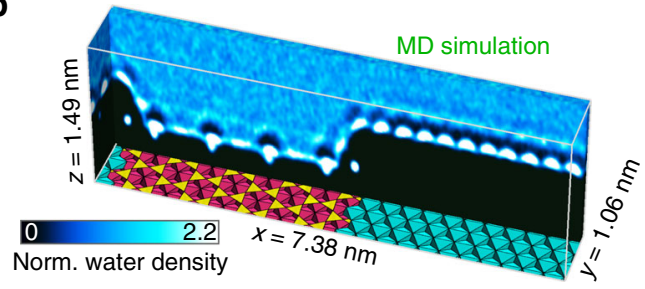

g

1st layer on Bı region

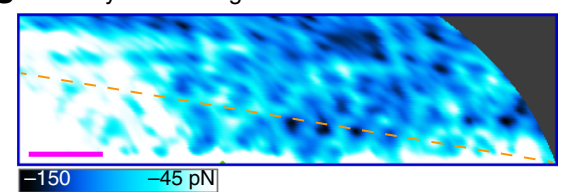

h 2nd layer on Bı region

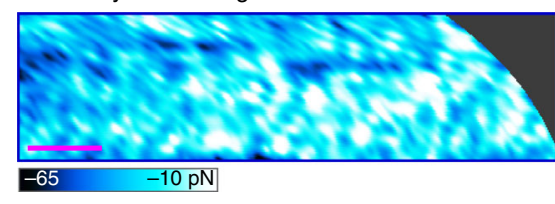

Experiment

m Perpendicular map on Bı region

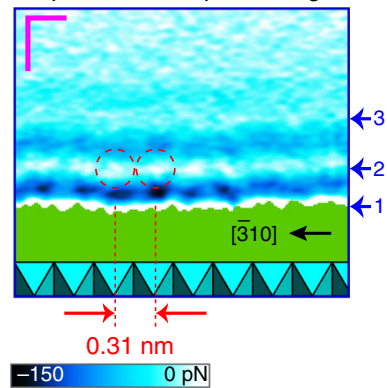

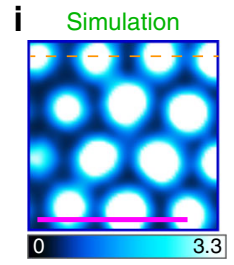

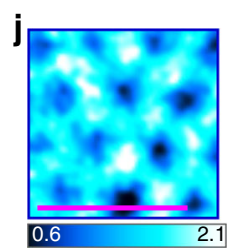

n

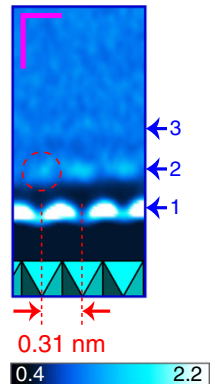

Fig. 2 Comparison of 3D FM-AFM and MD results on each terrace region. $\mathbf{a}$, b Representations of 3D force map (a) and MD simulation (b) on the clinochlore (001) surface. $\mathbf{c}, \mathbf{d}, \mathbf{g}, \mathbf{h}$ Lateral 2D force maps measured at the tip heights presumably corresponding to the first (c) and second (d) layers on the $T$ region, and the first $(\mathbf{g})$ and second $(\mathbf{h})$ layers on the B region. $\mathbf{e}, \mathbf{f}, \mathbf{i}, \mathbf{j}$ Theoretical lateral 2D-normalised water (oxygen) density maps of the first higher (e) and second layers (f) on the T region, and the first (i) and second layers (j) on the B region. $\mathbf{k}, \mathbf{m}$ Perpendicular 2D force maps along the broken lines in $\mathbf{c}$ on the T region ( $\mathbf{k}$ ), and $\mathbf{g}$ on the $B_{1}$ region $(\mathbf{m})$. I, $\mathbf{n}$ Theoretical perpendicular 2D-normalised water (oxygen) density maps along the broken lines in $\mathbf{e}$ on the $T$ region ( $\mathbf{I}$, and $\mathbf{i}$ on the B region ( $\mathbf{n}$ ). The adsorbed first lower layer in the centre of the honeycomb and the first higher layer forming the honeycomb-like pattern are shown as $1_{\mathrm{L}}$ and $1_{\mathrm{H}}$, respectively. The red broken circles indicate the periodic hydration structures. Zero-distances in the simulations are defined as the centres of the oxygen atoms of each surface. Scale bars: $0.8 \mathrm{~nm}(\mathbf{c}-\mathbf{j})$ and $0.3 \mathrm{~nm}(\mathbf{k}-\mathbf{n})$

surface charge density on the $\mathrm{B}_{\mathrm{II}}$ region was $+0.05 \mathrm{C} / \mathrm{m}^{2}$ (Supplementary Note 2), which was significantly lower than that on the $B_{I}$ region due to the edge effect as discussed later. For the short-range force observed on the $\mathrm{T}$ regions, we consider that it was possibly due to the relaxation of the tip/sample atoms, as previous theoretical studies on the interactions between the positive/negative tip on the positive/negative ions of calcite in solutions showed different tip relaxation behaviours depending on the tip/sample polarities ${ }^{17,36}$. We also consider that the shortrange repulsive force could be due to strongly bound cations in the hollow sites, as we also observed a similar short-range repulsive force on muscovite mica in a high-salt solution ${ }^{13}$. Since we cannot determine the tip configuration during the experiment, identifying the role of the tip would require a very extensive set of simulations including free-energy calculations using various tip/ sample models and more experiments to identify the origin of the short-range force. This is an ongoing challenge for the field, but is beyond the scope of the current work.
We retrieved the normalised water density curves (averaged over the entire surfaces) from the MD simulations (Fig. 3c, d). When comparing the hydration peak distances, we consistently observed that there was no intrinsic difference in the hydration layer spacing for either the $\mathrm{T}$ or $\mathrm{B}_{\mathrm{I}}$ regions; namely, 0.31 and $0.30 \mathrm{~nm}$ in the experiments, and 0.25 and $0.24 \mathrm{~nm}$ in the simulation for the $\mathrm{T}$ and $\mathrm{B}_{\mathrm{I}}$ regions, respectively (see arrows in Fig. 3). Although the experimental forces and theoretical water densities have many features in common, in principle, we cannot compare them directly ${ }^{17}$. To overcome this problem, we also converted the densities to force by the recently proposed solvent tip approximation model ${ }^{39}$, and we obtained consistent results regarding the terrace regions (Supplementary Note 3).

Intermediate region around the step edge. From the $3 \mathrm{D}$ FMAFM data, we reconstructed a topographic image of the clinochlore (001) surface (Fig. 4a), whose model is shown in Fig. 4b. 

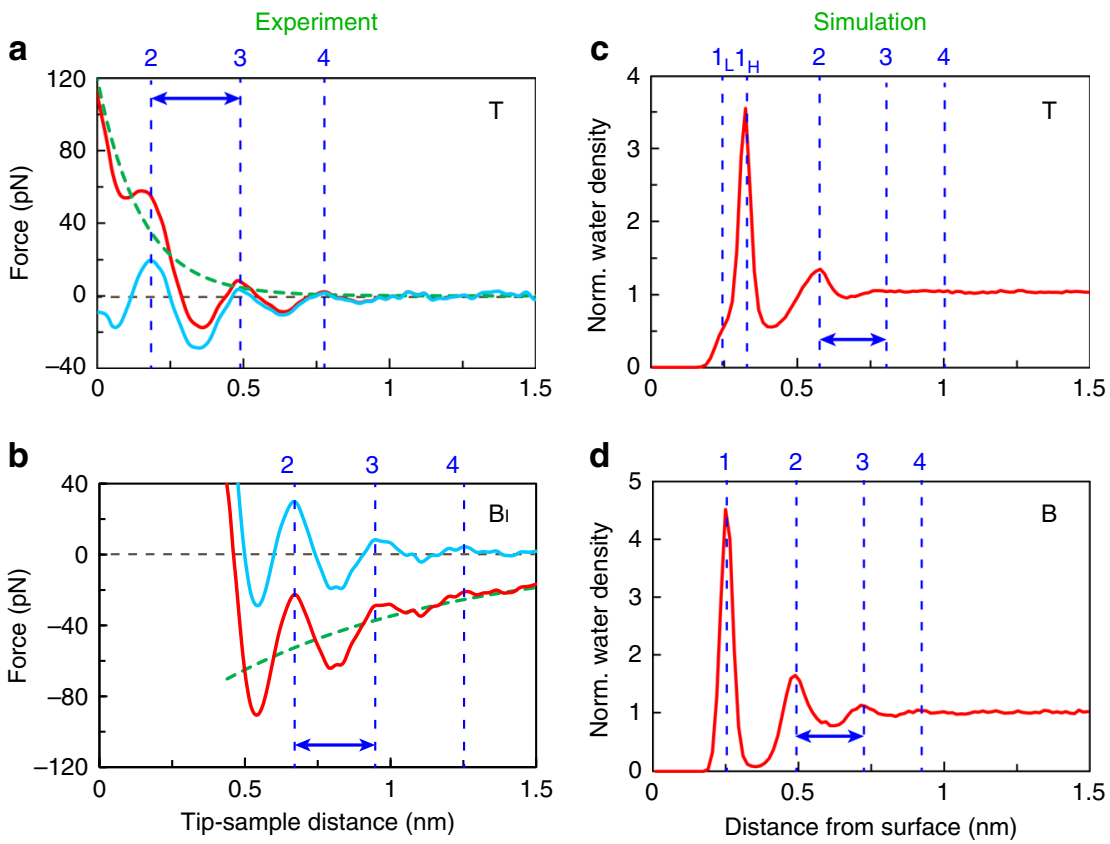

Fig. 3 Comparison of force curves and density profiles. $\mathbf{a}$, b Average force profiles on the $T(\mathbf{a})$ and $B_{1}(\mathbf{b})$ regions extracted from 3D force map (red curves). The broken green and solid light-blue curves are the fitted background force and subtracted force curves, respectively. The vertical blue broken lines represent the force maxima in the subtracted curves. $\mathbf{c}, \mathbf{d}$ Normalised water (oxygen) and ion density profiles calculated by MD simulation on the T (c) and $B(d)$ regions

Along the broken lines $\mathrm{P}-\mathrm{Q}$ and $\mathrm{R}-\mathrm{S}$ (both running in the $[\overline{3} 10]$ direction), we extracted the $2 \mathrm{D}$ force maps (Fig. $4 \mathrm{c}$ ). On the $\mathrm{B}_{\mathrm{II}}$ region, we did not observe the dot-like patterns, which was consistent with the result that atomic-resolution imaging on the $B_{I I}$ region was less clear than that on the $B_{I}$ region. As the apparent height of the $B_{I I}$ region was about $0.3 \mathrm{~nm}$, we conclude that the tip was scanned over the first layers on the $T$ and $B_{I}$ regions, giving the atomic resolution on both the regions, while the tip was directly scanned over the solid surface in the $B_{\text {II }}$ region.

\section{Discussion}

We show 2D theoretical water (oxygen) and ion density maps in Fig. $5 \mathrm{a}, \mathrm{b}$, which are presented with the unit of $\mathrm{mol} / \mathrm{L}$ and not normalised to the bulk density. Note that we used a $1 \mathrm{M}$ solution in the initial state due to time and size constraints (see Supplementary Fig. $6 \mathrm{c}$ for the snapshot), which had a ten times higher ion concentration than the experimental condition. Under this condition, the water density is still orders of magnitude larger than the ion density even in the vicinity of the charged surfaces. We consider that the ions were moving around during the millisecond timescale of the AFM measurements, and thus that the experimentally observed hydration forces and their contrast patterns in the 2D maps mainly originate from the water density distribution. This is supported by repeated observations of the common similarities between experimental force and simulated water/force on several other surfaces ${ }^{13}, 14,17,21,23,31-35$. A recent paper on FM-AFM measurements of the hydration structures of calcium fluorite in ultra-pure water and supersaturated solution has shown that the adsorbed ions could give a slight modulation in the oscillatory force profiles, but they did not change the overall features ${ }^{33}$. Actually, we also observed the hydration structures on muscovite mica in ultra-pure water as well as in high-salt solutions (Supplementary Note 4). We do not deny the role of ions in the AFM measurement, but their effects are limited. For the $\mathrm{T}$ region, Fig. $5 \mathrm{~d}$ shows that the innermost cations a

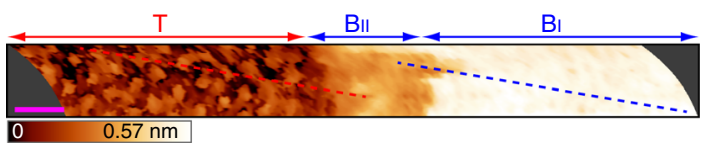

b
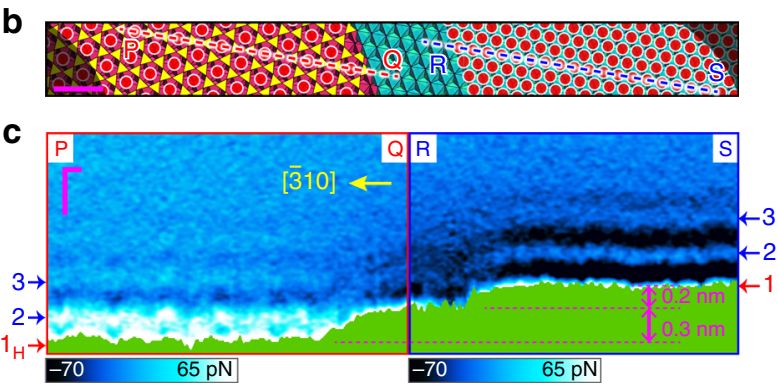

Fig. 4 Experimental local hydrations around the step edge. $\mathbf{a}, \mathbf{b}$ Topographic image (a) and structural model (b) of the clinochlore (001) surface reconstructed from 3D $\Delta f$ data $(\Delta f=290 \mathrm{~Hz})$. Red dots represent the adsorbed water molecules on the first layers on the $T$ and $B_{1}$ regions. $c$ Perpendicular 2D force maps extracted from the 3D force data along the broken lines $\mathrm{P}-\mathrm{Q}$ and $\mathrm{R}-\mathrm{S}$ in $\mathbf{a}$. Scale bars: $1 \mathrm{~nm}(\mathbf{a}, \mathbf{b})$ and $0.3 \mathrm{~nm}(\mathbf{c})$

$\left(\mathrm{Na}^{+}\right)$are located closer to the surface than the oxygens of the water molecules. As already mentioned, the short-range repulsive force observed on the $T$ region could be because of these strongly bound cations in the hollow sites. On the other hand, for the B region, we see in Fig. 5d that the innermost anions are competing with the water oxygens at almost the same height, and they create the opposite patterns (see the right side of Fig. 5a, c). As previously mentioned, the ambiguous or possibly inverted $2 \mathrm{D}$ patterns observed on the $\mathrm{B}$ region could be due to the anions $\left(\mathrm{Cl}^{-}\right)$. This result is consistent with the experimental result that we observed, the long-range electrostatic force on the $\mathrm{B}$ region but not on the $\mathrm{T}$ layer probably because most of the negative charges 

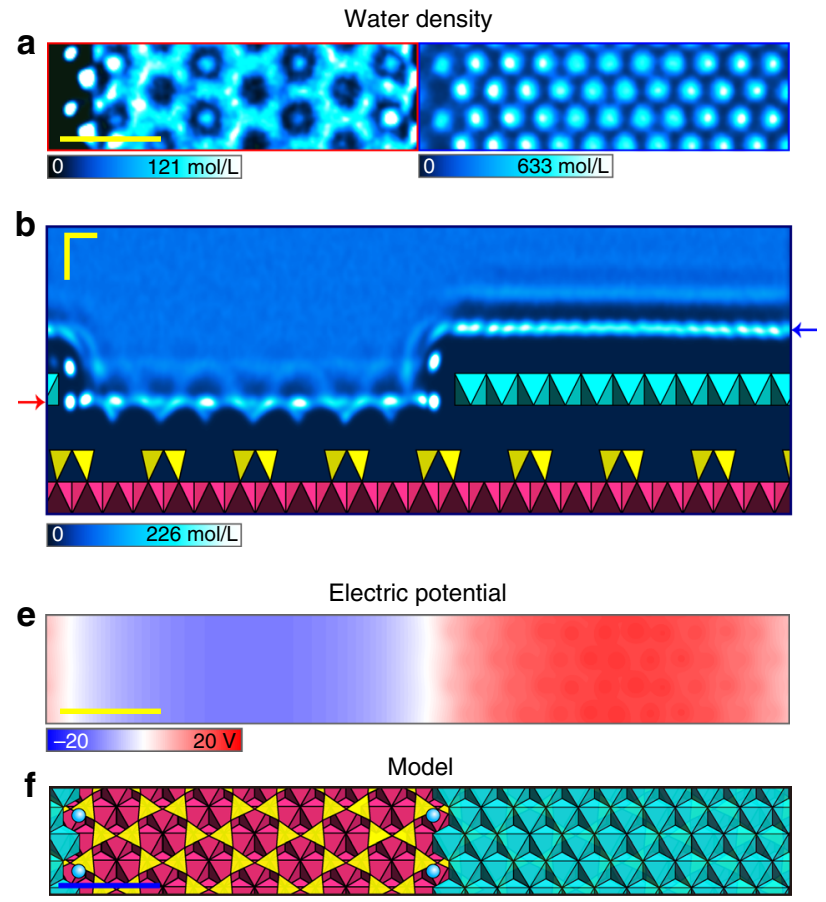

c

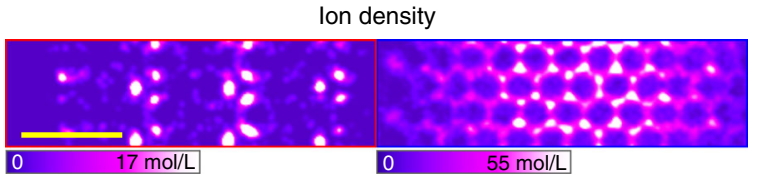

d

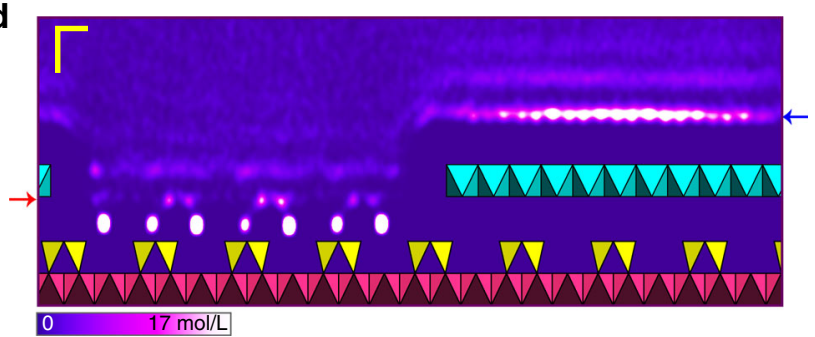

g

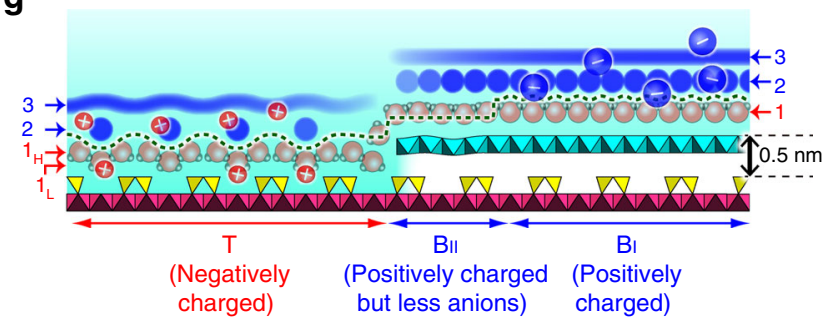

Fig. 5 Theoretical local hydrations and ions around the step edge. a, c Lateral water (oxygen) (a) and ion (c) density maps from the MD simulation in the first layers on the $T$ and $B$ regions. $\mathbf{b}, \mathbf{d}$ Perpendicular water (b) and ion (d) density maps averaged along the [001] direction, where the arrows indicate the heights of the lateral water density maps in $\mathbf{a}$ and $\mathbf{c}$, respectively. $\mathbf{e}$ Electric potential of $0.1 \mathrm{~nm}$ above the outermost oxygen atoms of the $B$ region (showing a zero potential at the step edge, in white). $\mathbf{f}$ Structural model used in the MD simulation. $\mathbf{g}$ Schematic illustration of the cross section of the clinochlore (001) surface along the dashed lines in Fig. 4a, b. The green dashed curve shows the apparent surface (trajectory of the tip scan during imaging topography). Scale bars: $1.0 \mathrm{~nm}(\mathbf{a}, \mathbf{c}, \mathbf{e})$ and $0.3 \mathrm{~nm}(\mathbf{b}, \mathbf{d})$

on the $\mathrm{T}$ region are screened by the cations inside the hydration layer. Finally, it should also be noted that several researchers have resolved individual adsorbed ions by AFM if they strongly bound on the surfaces with the timescale longer than the AFM measurements $^{22,40}$.

The MD simulations also suggest a reason why no clear hydration structures could be observed in the $\mathrm{B}_{\mathrm{II}}$ region. Around a step edge, the water densities in the first layers (top view, Fig. 5a, and side view, Fig. 5b) indeed show an increase, while the ion (cation and anion) densities (top view, Fig. 5c, and side view, Fig. 5d) are decreased. The orientations of the water molecules around the step edges were comparable to those on the terrace, meaning that this depletion of ions is a possible candidate for the indistinct hydration structure. It has been experimentally shown that a high ion concentration often enhances the image contrast of the hydration structure ${ }^{12-16}$, which can be explained by the reduction in the diffusion coefficient and the increase in the residence time of the water molecules 24,41 .

In order to discuss the origin of the depletion, we calculated the electric potential as shown in Fig. 5e (see Supplementary Methods for the details). As a result, we found a neutral area around the step edge, and the potential on the B layer was reduced in the vicinity of the step edges. In the case of an electrified metal, where the boundary condition is the constant-potential, the electric fields and ion concentration around edge structures are enhanced due to the edge effect ${ }^{3}$. On the other hand, in the case of a fully charged system such as the B layer, where the boundary condition is a constant-charge, the electric fields around the edges are the same as those on the flat surfaces, while the surface potentials and ion concentration around the edges significantly decrease. The reduced surface potential causes the depletion of ions, while the constant electric field maintains the same hydration structure as that on the terrace. Increasing the ion concentration reduced the width of the $\mathrm{B}_{\mathrm{II}}$ region (Supplementary Fig. 2), which supports this explanation.

A schematic illustration of the cross section of the clinochlore (001) step deduced from the experiments and simulations is shown in Fig. 5g. We explicitly draw the water molecules in the first layers and use blue patterns as the upper hydration layers observed by liquid-3D FM-AFM. We only observed the multiple hydration layers on the $\mathrm{T}$ and $\mathrm{B}_{\mathrm{I}}$ regions, but barely observed any layers on the $\mathrm{B}_{\mathrm{II}}$ region although there are hydration layers on the $\mathrm{B}_{\mathrm{II}}$ region as well. Our results clearly show that the liquid-3D FMAFM can distinguish the local hydration structures reflecting different surface properties even at the domain boundaries.

In summary, we performed the 3D FM-AFM experiment and MD simulations on the clinochlore (001) surface in aqueous solution. By comparing these results, we conclude that lattice periodicities are reflected in the intrinsic lateral water distributions, which are experimentally observed as a laterally periodic hydration structure. Meanwhile, the surface charge difference is reflected in the adsorption behaviour of the ion species, which are experimentally observed as the long-range background force. We also discovered intermediate regions at the step edges of the $\mathrm{B}$ islands, which likely originate from ion depletion by the edge effect, although the stability of different step morphologies could also play a role.

This work is, to the best of our knowledge, the first demonstration of the 3D FM-AFM measurements on an inhomogeneous mineral surface since 3D FM-AFM has so far been applied mainly to atomically flat homogeneous mineral surfaces ${ }^{12-15}, 17,32-34$. This was quite challenging because it has step-and-terrace structures and the oppositely charged areas. However, we developed a fast and nondestructive acquisition protocol by disabling 
the constant frequency shift feedback and setting the frequency threshold instead, which enabled us to acquire the 3D map on an uneven heterogeneous mineral surface. We consider this as an important milestone in the development of the FM-AFM/MD method, and we hope that the methodology, once established, could be applicable for the hydration structures of more complicated systems such as catalysis, electrochemistry and even biological molecules including proteins and nucleic acids in the future. Here, we also remark that the clinochlore (001) surface can also serve as an experimental platform for exploring the biological processes because it can be used for patterning ${ }^{27}$ or suspension (Supplementary Note 5) of biological molecules. Since FM-AFM could give some information on the outer hydration shells surrounding biomolecules in vivo, we consider that it is useful as a complementary tool to the conventional techniques that mainly give the information on the distribution of the water molecules inside the biomolecules.

\section{Methods}

FM-AFM setup. We used a customised commercial AFM head (Shimadzu: SPM9600) with a homebuilt digital PXI controller (National Instruments: NI PXI-8196) based on a high-speed field-programmable gate array (FPGA) board (National Instruments: NI PXI-7833R) programmed by LabVIEW (National Instruments) and a homebuilt FM detector circuit ${ }^{43}$. In order to achieve quantitative and reproducible force measurements in a liquid environment, we employed a photothermal excitation setup ${ }^{44}$. We used a rectangular cantilever with a gold backside coating (Nanosensors: PPP-NCHAuD), whose nominal spring constant $\left(k_{z}\right)$ was $42 \mathrm{~N} / \mathrm{m}$. The $k_{z}$ of the cantilevers used in the experiments was determined to be 35 $\mathrm{N} / \mathrm{m}$ by Sader's method ${ }^{45}$. The resonance frequency of the cantilever $\left(f_{0}\right)$ was 131 $\mathrm{kHz}$ in a $100 \mathrm{mM} \mathrm{KCl}$ solution. Immediately prior to each experiment, organic contaminations on the tip were removed by irradiating the tip using a UV-ozone cleaner (Filgen: UV253) for a few hours.

The measurement was performed in $100 \mathrm{mM}$ potassium chloride aqueous solutions $(\mathrm{KCl}, 99.5 \%$ purity, Wako Pure Chemical Industries, Ltd.) whose Debye length is $0.97 \mathrm{~nm}$ at $298 \mathrm{~K}$. This reagent was used without further purification and any $\mathrm{pH}$ regulation. The aqueous solution was slightly acidified to a $\mathrm{pH}$ value of around 5.7 due to the dissolved $\mathrm{CO}_{2}$ gas. All the experiments are conducted in a temperature-regulated enclosure (Mitsubishi Electric Engineering Company, Ltd.: CN-40A), which can maintain a constant temperature of $298 \pm 0.1 \mathrm{~K}$ and thus reduce the influence of thermal drift by the AFM head.

3D FM-AFM measurement. The 3D map measurement on an uneven heterogeneous surface was realised by the real-time monitoring ${ }^{13,25}$, instead of a timeaveraging monitoring ${ }^{12}$, of the frequency shift, which was implemented by a highspeed FPGA board. The signal-to-noise ratio and the acquisition time of the 3D map measurements are in a trade-off relation. When the scanning speed is reduced, the risk of surface deformation decreases, but the thermal drift increases. Conversely, when the scanning speed is increased, the thermal drift is reduced, but the surface deformation can occur. In order to keep a high-enough signal-to-noise ratio while suppressing the thermal drift, we need several improvements to the FM-AFM experiment system as follows.

A $3 \mathrm{D} \Delta f$ map with dimensions of $12.8 \times 1.5 \times 1.7 \mathrm{~nm}^{3}(256 \times 15 \times 173$ pixels $)$ was acquired by collecting perpendicular 2D $\Delta f$ maps (XZ slices). During the $2 \mathrm{D} \Delta f$ map acquisition, the frequency shift vs. distance curves (1D $\Delta f$ curves) were recorded by translating the tip towards the sample using a sawtooth waveform signal of $22 \mathrm{~Hz}$, which corresponds to a tip velocity of about $40 \mathrm{~nm} / \mathrm{s}$. The approach was immediately stopped when the frequency shift signal reached a predetermined threshold value, and then the tip was retracted to the original position.

Proper setting of the threshold value minimises the possibility of the tip change as well as the sample damage, and thereby secures the continuity of the consecutively recorded force curves. By omitting the retraction curve measurement, the total acquisition time can be reduced by almost half to $1.5 \mathrm{~min}$. Although it has been demonstrated that a 3D $\Delta f$ map can be collected by employing a sinusoidal tip motion with a slow constant frequency shift feedback ${ }^{12,14,16-18,32,33}$, it is not suitable for acquiring a $3 \mathrm{D} \Delta f$ map on an uneven heterogeneous surface, where large variations in the topographic height and long-range electric double-layer force are expected during the data acquisition.

In ultra-high vacuum (UHV), the atom-tracking technique is often used for suppressing the thermal driff ${ }^{46}$. However, in a liquid, it is difficult to implement because the thermal drift is nonlinear and the tip change often occurs due to the higher interaction force than that in the UHV. In order to solve this problem, we used a real-time feedforward system. Additionally, an automatic post-processing programme is also crucial for the efficient time-consuming 3D map measurements.

As described above, the photothermal excitation setup ${ }^{44}$ is essential for stabilising the uncontrollable fluctuation of the resonance frequency. The preparation method for the tip and sample is also essential for high-resolution 3D map imaging. Any organic contaminants on the tip should be removed by a UVozone cleaner immediately before each experiment for avoiding unstable tip conditions. A droplet of the solution should be placed on the sample as soon as possible after the cleavage of the sample for avoiding any contamination. Since an adhesive for fixing the sample to a magnetised stainless-steel plate often contaminates the solution, a larger sample size should be used in order to cover the entire stainless-steel plate.

Post-processing of 3D FM-AFM data. We established an automatic postprocessing programme based on Visual Studio (Microsoft), which is essential for efficient analysis of the large-size 3D map data. First, the 3D $\Delta f$ data were smoothed by using a Gaussian filter with a standard deviation of $0.034 \mathrm{~nm}(x$ and $y$ ) and $0.011 \mathrm{~nm}(z)$, and the 3D force data were obtained by converting each $2 \mathrm{D} \Delta f$ map to a $2 \mathrm{D}$ force map using Sader's method ${ }^{47}$. Since the background offset of the 1D curves in the 2D map contained fluctuations, they were smoothed using a Gaussian filter with a standard deviation of $1.23 \mathrm{~nm}$ before and after the conversion. Second, the resolution of the $3 \mathrm{D}$ data was increased to $1024 \times 113 \times 346$ pixels using a Lanczos interpolation filter of an order of 3 (Lanczos-3) ${ }^{48}$. Third, the background offset in each force curve was corrected using an analytical formula based on the electric double-layer theory ${ }^{25}$. Note that the force curve in Fig. $3 \mathrm{~b}$ has a non-zero value in the farthest part because of the offset correction. Finally, the linear and nonlinear lateral drifts were corrected such that the lattice constants in the $2 \mathrm{D}$ lateral maps match those in the literature ${ }^{49}$. For the $2 \mathrm{D}$ force maps presented in Figs. $2 \mathrm{k}, \mathrm{m}$ and $4 \mathrm{c}$, the boundaries between the pixels with data (blue colour) and without data (green colour) were interpolated using the Lanczos-3 filter

MD simulations. All the MD simulations were performed using LAMMPS (LargeScale Atomic/Molecular Massively Parallel Simulator) $\operatorname{code}^{50}$ under ambient conditions ( $300 \mathrm{~K}$ and 1 bar) using a Nosé-Hoover scheme and a time step of $1 \mathrm{fs}$. Along all the dimensions, periodic boundary conditions were employed. The surfaces were first optimised in vacuum in order to remove any unphysical interactions between overlapping atoms and then immersed into water. After water equilibration (for $25 \mathrm{ps}$ ), all atoms in the crystal were allowed to move, except for the $\mathrm{Mg}$ atoms in the central layer (in order to prevent drift of the entire system), and the system was equilibrated again for $25 \mathrm{ps}$. The target pressure was specified only along the $z$ direction (to protect the crystal from lateral stress) allowing the simulation box to change during the dynamics, ensuring the proper water density in the bulk phase. The production MD runs with flat terraces were only performed for $10 \mathrm{~ns}$, but it was increased to $15 \mathrm{~ns}$ for the systems with a clinochlore step present. This longer simulation time was necessary to allow ions in the solution to sufficiently diffuse in order to be able to reach the surface or step edge.

We obtained the crystal structure of clinochlore from the American Mineralogist Crystal Structure Database ${ }^{49}$. First, we simulated the hydration structures exposed on each region as shown in Fig. 2 (see Supplementary Fig. 6a, b for the snapshots). Based on the unit cell data, we constructed a simulation cell with lateral dimensions in the (001) plane of roughly $2.1 \times 3.7 \mathrm{~nm}^{2}$. In the [001] direction (in terms of our Cartesian simulation cell, this is the $z$ direction), we repeated sufficient layers of clinochlore to make sure that the internal part of the crystal is under no additional stress caused by the nearby free surfaces. A typical system contained $\sim 2800$ atoms for the clinochlore crystal and over 3000 water molecules.

Next, we simulated the hydration structures exposed on a step region as shown in Fig. 5 (see Supplementary Fig. $6 c$ for the snapshot). In this case, we cleaved the surface along the (001) plane and removed one-half of the B layer to form the step edge running in the [100] direction, thus exposing the underlying layer as the lower terrace. In order to avoid a lateral dipole effect on the step edge, we created a larger system with the lateral dimension of $4.3 \times 7.4 \mathrm{~nm}^{2}$. Step edges created this way were neutral. In order to investigate all possible edges that might appear in the real system, we modelled another step edge by cutting the B layer along the zigzag line (Supplementary Note 6). This way, we have created a step that matches triangular structures seen in experiments. This type of step edge was negatively charged due to the protruding oxygen atoms along the edge. In order to maintain a neutral system, an opposite triangle pit was introduced, consisting of exposed metal ions and therefore positively charged. In general, MD simulations showed that the triangular systems were not stable, with significant desorption of $\mathrm{OH}$. Hence, we decided to focus on the stable (neutral) step edges in our simulations, but the results for the other steps are discussed in Supplementary Note 6. On either side of the clinochlore sample, water molecules were placed to solvate the system and to make sure that far away from the solid-liquid interfaces, water behaves as in the bulk. The system with the exposed step contained 4600 crystal atoms and $~ 13,000$ water molecules.

All interactions between the atoms were described by two force fields. The CLAYFF force field ${ }^{51}$ was used to describe all interactions between the clinochlore atoms, water and ions present in solution. This force field has been successfully used in the past in many clay mineral MD simulations ${ }^{24,52,53}$. Although CLAYFF force field was developed to fully describe the properties of clay minerals, small modifications $(<3 \%)$ of the partial charges were required in order to maintain an electrostatically neutral system for the surfaces and steps considered here (octahedral magnesium in the B layer +1.37 new/ +1.36 CLAYFF; bridging oxygen 
with tetrahedral substitution in the T layer $-1.16875 /-1.1688$ and hydroxyl oxygen in the B layer $-0.9775 /-0.95)$. However, these small deviations are consistent with charge-balancing approach in CLAYFF. As a water model in our simulations, we used the flexible TIP $3 \mathrm{P}^{54}$ model. All parameters for this model were optimised to reproduce experimental thermodynamic and structural properties of liquid water as well as vibrational spectra. We also checked all simulations using the nonbonded three-body harmonic potential energy term for $\mathrm{Mg}-\mathrm{O}-\mathrm{H}$ interactions ${ }^{55}$ and modified charges ${ }^{56}$. These made no significant difference to our results on the standard step edge. However, the included nonbonded three-body term for $\mathrm{Mg}-\mathrm{O}-\mathrm{H}$ bending interactions significantly improved the stability of the triangle step structure (changing the charges did not), but not fully, as we still observed the desorption of some hydroxyl groups during simulations.

For visual inspection, VMD ${ }^{57}$ was used and most of the analysis was performed using the Python library MDAnalysis ${ }^{58}$. The $2 \mathrm{D}$ water density maps presented in Figs. 2 and 5 were created after smoothing the time-averaged 3D trajectory data using a Gaussian kernel filter with a standard deviation of $0.021 \mathrm{~nm}$ in all three directions and a linear interpolation.

Data availability. The data that support the findings of this study are available from the corresponding authors upon reasonable request.

Received: 17 March 2017 Accepted: 24 October 2017

Published online: 13 december 2017

\section{References}

1. Raccichini, R., Varzi, A., Passerini, S. \& Scrosati, B. The role of graphene for electrochemical energy storage. Nat. Mater. 14, 271-279 (2015).

2. Holewinski, A., Idrobo, J. C. \& Linic, S. High-performance Ag-Co alloy catalysts for electrochemical oxygen reduction. Nat. Chem. 6, 828-834 (2014).

3. Liu, M. et al. Enhanced electrocatalytic $\mathrm{CO}_{2}$ reduction via field-induced reagent concentration. Nature 537, 382-386 (2016).

4. Deng, D. H. et al. Catalysis with two-dimensional materials and their heterostructures. Nat. Nanotechnol. 11, 218-230 (2016).

5. Croce, R. \& van Amerongen, H. Natural strategies for photosynthetic light harvesting. Nat. Chem. Biol. 10, 492-501 (2014).

6. Mukai, $\mathrm{H}$. et al. Cesium adsorption/desorption behavior of clay minerals considering actual contamination conditions in Fukushima. Sci. Rep. 6, 21543 (2016).

7. Buurmans, I. L. C. \& Weckhuysen, B. M. Heterogeneities of individual catalyst particles in space and time as monitored by spectroscopy. Nat. Chem. $\mathbf{4}$, 873-886 (2012)

8. Niimura, N. \& Podjarny, A. Neutron Protein Crystallography: Hydrogen, Protons, and Hydration in Bio-macromolecules (Oxford University Press, Oxford, UK, 2011).

9. Merk, A. et al. Breaking cryo-EM resolution barriers to facilitate drug discovery. Cell 165, 1698-1707 (2016).

10. Daillant, J. \& Gibaud, A. X-ray and Neutron Reflectivity: Principles and Applications (Springer, Berlin, Germany, 2009).

11. Israelachvili, J. N. Intermolecular and Surface Forces, 3rd ed (Academic Press, Cambridge, MA, 2010).

12. Fukuma, T., Ueda, Y., Yoshioka, S. \& Asakawa, H. Atomic-scale distribution of water molecules at the mica-water interface visualized by three-dimensional scanning force microscopy. Phys. Rev. Lett. 104, 016101 (2010).

13. Kobayashi, K. et al. Visualization of hydration layers on muscovite mica in aqueous solution by frequency-modulation atomic force microscopy. J. Chem. Phys. 138, 184704 (2013).

14. Martin-Jimenez, D., Chacon, E., Tarazona, P. \& Garcia, R. Atomically resolved three-dimensional structures of electrolyte aqueous solutions near a solid surface. Nat. Commun. 7, 12164 (2016).

15. Labuda, A., Kobayashi, K., Suzuki, K., Yamada, H. \& Grütter, P. Monotonic damping in nanoscopic hydration experiments. Phys. Rev. Lett. 110, 066102 (2013).

16. Asakawa, H., Yoshioka, S., Nishimura, K. \& Fukuma, T. Spatial distribution of lipid headgroups and water molecules at membrane/water interfaces visualized by Three-Dimensional scanning force microscopy. ACS Nano 6, 9013-9020 (2012).

17. Fukuma, T. et al. Mechanism of atomic force microscopy imaging of threedimensional hydration structures at a solid-liquid interface. Phys. Rev. B 92 155412 (2015).

18. Herruzo, E. T., Asakawa, H., Fukuma, T. \& Garcia, R. Three-dimensional quantitative force maps in liquid with 10 piconewton, angstrom and subminute resolutions. Nanoscale 5, 2678-2685 (2013).

19. Reischl, B., Watkins, M. \& Foster, A. S. Free energy approaches for modeling atomic force microscopy in liquids. J. Chem. Theory Comput. 9, 600-608 (2013).

20. Hofmann, S., Voitchovsky, K., Spijker, P., Schmidt, M. \& Stumpf, T. Visualising the molecular alteration of the calcite (104)-water interface by sodium nitrate. Sci. Rep. 6, 21576 (2016).
21. Spijker, P. et al. Understanding the interface of liquids with an organic crystal surface from atonnistic simulations and AFM experiments. J. Phys. Chem. C 118, 2058-2066 (2014).

22. Ricci, M., Spijker, P. \& Voitchovsky, K. Water-induced correlation between single ions imaged at the solid-liquid interface. Nat. Commun. 5, 4400 (2014).

23. Ito, F. et al. Molecular resolution of the water interface at an alkali halide with terraces and steps. J. Phys. Chem. C 120, 19714-19722 (2016).

24. Wang, J. W., Kalinichev, A. G. \& Kirkpatrick, R. J. Effects of substrate structure and composition on the structure, dynamics, and energetics of water at mineral surfaces: a molecular dynamics modeling study. Geochim. Cosmochim. Acta 70, 562-582 (2006)

25. Umeda, K., Kobayashi, K., Oyabu, N., Matsushige, K. \& Yamada, H. Molecularscale quantitative charge density measurement of biological molecule by frequency modulation atomic force microscopy in aqueous solutions. Nanotechnology 26, 285103 (2015).

26. Vrdoljak, G. A., Henderson, G. S., Fawcett, J. J. \& Wicks, F. J. Structural relaxation of the chlorite surface imaged by the atomic-force microscope. Am. Mineral. 79, 107-112 (1994).

27. Antognozzi, M. et al. A chlorite mineral surface actively drives the deposition of DNA molecules in stretched conformations. Nanotechnology 17, 3897-3902 (2006).

28. Valdrè, G., Moro, D., Hounsome, C. M. \& Antognozzi, M. SPM nanolithography of hydroxy-silicates. Nanotechnology 23, 385301 (2012).

29. Gazzè, S. A., Stack, A. G., Ragnarsdottir, K. V. \& McMaster, T. J. Chlorite topography and dissolution of the interlayer studied with atomic force microscopy. Am. Mineral. 99, 128-138 (2014).

30. Valdrè, G., Malferrari, D. \& Brigatti, M. F. Crystallographic features and cleavage nanomorphology of chlinochlore: specific applications. Clays Clay Miner. 57, 183-193 (2009).

31. Miyata, K. et al. Dissolution processes at step edges of calcite in water investigated by high-speed frequency modulation atomic force microscopy and simulation. Nano Lett. 17, 4083-4089 (2017).

32. Miyazawa, K. et al. A relationship between three-dimensional surface hydration structures and force distribution measured by atomic force microscopy. Nanoscale 8, 7334-7342 (2016)

33. Miyazawa, K., Watkins, M., Shluger, A. L. \& Fukuma, T. Influence of ions on two-dimensional and three-dimensional atomic force microscopy at fluoritewater interfaces. Nanotechnology 28, 245701 (2017).

34. Söngen, H. et al. Chemical identification at the solid-liquid interface. Langmuir 33, 125-129 (2017).

35. Araki, Y. et al. Direct observation of the influence of additives on calcite hydration by frequency modulation atomic force microscopy. Cryst. Growth Des. 14, 6254-6260 (2014)

36. Tracey, J. et al. Understanding $2 \mathrm{D}$ atomic resolution imaging of the calcite surface in water by frequency modulation atomic force microscopy. Nanotechnology 27, 415709 (2016).

37. Pashley, R. M. DLVO and hydration forces between mica surfaces in $\mathrm{Li}^{+}, \mathrm{Na}^{+}$ $\mathrm{K}^{+}$and $\mathrm{Cs}^{+}$electrolyte solutions: a correlation of double-layer and hydration forces with surface cation exchange properties. J. Colloid Interface Sci. 83, 531-546 (1981)

38. Jiao, Y. N. \& Hou, W. G. Effects of structural charges on points of zero charge and intrinsic surface reaction equilibrium constants of $\mathrm{Zn}-\mathrm{Al}$ and $\mathrm{Zn}-\mathrm{Al}-\mathrm{Fe}$ hydrotalcite-like compounds. Colloids Surf. A: Physicochem. Eng. Aspects 296, 62-66 (2007)

39. Watkins, M. \& Reischl, B. A simple approximation for forces exerted on an AFM tip in liquid. J. Chem. Phys. 138, 154703 (2013).

40. Siretanu, I., van den Ende, D. \& Mugele, F. Atomic structure and surface defects at mineral-water interfaces probed by in situ atomic force microscopy. Nanoscale 8, 8220-8227 (2016).

41. Du, H. \& Miller, J. D. Interfacial water structure and surface charge of selected alkali chloride salt crystals in saturated solutions: a molecular dynamics modeling study. J. Phys. Chem. C 111, 10013-10022 (2007).

42. Momma, K. \& Izumi, F. VESTA 3 for three-dimensional visualization of crystal, volumetric and morphology data. J. Appl. Crystallogr. 44, 1272-1276 (2011).

43. Kobayashi, K., Yamada, H., Itoh, H., Horiuchi, T. \& Matsushige, K. Analog frequency modulation detector for dynamic force microscopy. Rev. Sci. Instrum. 72, 4383-4387 (2001).

44. Kobayashi, K., Yamada, H. \& Matsushige, K. Reduction of frequency noise and frequency shift by phase shifting elements in frequency modulation atomic force microscopy. Rev. Sci. Instrum. 82, 033702 (2011).

45. Sader, J. E., Chon, J. W. M. \& Mulvaney, P. Calibration of rectangular atomic force microscope cantilevers. Rev. Sci. Instrum. 70, 3967-3969 (1999).

46. Sugimoto, Y. et al. Chemical identification of individual surface atoms by atomic force microscopy. Nature 446, 64-67 (2007).

47. Sader, J. E. \& Jarvis, S. P. Accurate formulas for interaction force and energy in frequency modulation force spectroscopy. Appl. Phys. Lett. 84, 1801-1803 (2004). 
48. Burger, W. \& Burge, M. J. Principles of Digital Image Processing: Core Algorithms (Springer, London, 2009).

49. Phillips, T. L., Loveless, J. K. \& Bailey, S. W. $\mathrm{Cr}^{3+}$ coordination in chlorites structural study of 10 chromian chlorites. Am. Mineral. 65, 112-122 (1980).

50. Plimpton, S. Fast parallel algorithms for short-range molecular-dynamics. J. Comp. Phys. 117, 1-19 (1995).

51. Cygan, R. T., Liang, J. J. \& Kalinichev, A. G. Molecular models of hydroxide, oxyhydroxide, and clay phases and the development of a general force field. $J$. Phys. Chem. B 108, 1255-1266 (2004).

52. Wang, J. W., Kalinichev, A. G. \& Kirkpatrick, R. J. Asymmetric hydrogen bonding and orientational ordering of water at hydrophobic and hydrophilic surfaces: a comparison of water/vapor, water/talc, and water/mica interfaces. $J$. Phys. Chem. C 113, 11077-11085 (2009).

53. Du, H. \& Miller, J. D. A molecular dynamics simulation study of water structure and adsorption states at talc surfaces. Int. J. Miner. Process. 84, 172-184 (2007).

54. Wallqvist, A. \& Mountain, R. D. Reviews in Computational Chemistry, Vol. 13 (Eds Lipkowitz, K. B. \& Boyd, D. B.) 183-247 (John Wiley \& Sons, Inc., Hoboken, NJ, 1999).

55. Zeitler, T. R., Greathouse, J. A., Gale, J. D. \& Cygan, R. T. Vibrational analysis of brucite surfaces and the development of an improved force field for molecular simulation of interfaces. J. Phys. Chem. C 118, 7946-7953 (2014).

56. $\mathrm{Yu}, \mathrm{K}$. A. \& Schmidt, J. R. Elucidating the crystal face- and hydrationdependent catalytic activity of hydrotalcites in biodiesel production. J. Phys. Chem. C 115, 1887-1898 (2011).

57. Humphrey, W., Dalke, A. \& Schulten, K. VMD: visual molecular dynamics. J. Mol. Graph. 14, 33-38 (1996).

58. Michaud-Agrawal, N., Denning, E. J., Woolf, T. B. \& Beckstein, O. Software news and updates MDAnalysis: a toolkit for the analysis of molecular dynamics simulations. J. Comput. Chem. 32, 2319-2327 (2011).

\section{Acknowledgements}

This work was supported by Grants-in-Aid for Scientific Research from the Ministry of Education, Culture, Sports, Science and Technology of Japan (Grant No. 24221008, 25286057 and 15K17467), Foundation Advanced Technology Institute and the Murata Science Foundation, as well as the Academy of Finland through its Centres of Excellence Program (project no. 915804) and the EU project PAMS (contract no. 610446). The authors acknowledge use of the CSC, Helsinki for computational resources. The authors would also like to thank Ryohei Kokawa, Masahiro Ohta and Kazuyuki Watanabe of
Shimadzu Corporation. K.U. would like to thank Taketoshi Minato of Kyoto University for fruitful discussions for the simulations.

\section{Author contributions}

K.U. performed the 3D FM-AFM imaging, and analysed the data. K.K. developed the AFM instruments and electronics. H.K. performed the FM-AFM imaging on bacteriorhodopsin. L.Z., J.R. and P.S. performed the MD simulations. A.S.F. supervised the MD simulations. H.Y. supervised the whole study especially the experimental side. All authors have discussed the results and been involved in writing the manuscript.

\section{Additional information}

Supplementary Information accompanies this paper at https://doi.org/10.1038/s41467017-01896-4.

Competing interests: The authors declare no competing financial interests.

Reprints and permission information is available online at http://npg.nature.com/ reprintsandpermissions/

Publisher's note: Springer Nature remains neutral with regard to jurisdictional claims in published maps and institutional affiliations.

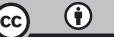

Open Access This article is licensed under a Creative Commons Attribution 4.0 International License, which permits use, sharing, adaptation, distribution and reproduction in any medium or format, as long as you give appropriate credit to the original author(s) and the source, provide a link to the Creative Commons license, and indicate if changes were made. The images or other third party material in this article are included in the article's Creative Commons license, unless indicated otherwise in a credit line to the material. If material is not included in the article's Creative Commons license and your intended use is not permitted by statutory regulation or exceeds the permitted use, you will need to obtain permission directly from the copyright holder. To view a copy of this license, visit http://creativecommons.org/ licenses/by/4.0/.

(C) The Author(s) 2017 